\title{
Schrödinger Operators with Moving Point Perturbations and Related Solvable Models of Quantum Mechanical Systems
}

\author{
V. A. Geyler and I. V. Chudaev
}

\begin{abstract}
A class of explicitly solvable models including those of low-dimensional systems in a magnetic field is considered. The spectral analysis of these models is reduced to the investigation of the spectrum of an ordinary differential operator perturbed by a point potential with varying support. The complete analysis of the variation of the eigenvalues and eigenfunctions with position of the potential support is given.
\end{abstract}

Keywords: Schrödinger operators, point perturbation, low-dimensional systems in a magnetic field, impurity in a well

AMS subject classification: $35 \mathrm{~J} 10$

\section{Introduction}

We start with an example of special interest to low-dimensional system physics. Consider a two-dimensional electron system [5] in the presence of a uniform magnetic field parallel to the system plane. The problem to describe the spectrum of the system arises in studies of the optical and transport properties of two-dimensional charge carriers subjected to a uniform in-plane magnetic field (see, e.c., $[3-5,8,14,20,23,33]$ ). The most frequently used picture of the spectrum is that obtained by means of a perturbation with respect to the field strength. Unfortunately, such perturbation methods are satisfactory in a rather restricted region of weak fields only [5]. The reason is that an arbitrary small variation of the field strength leads to an arbitrarily large variation of the vector potential of the field. In fact, the Hamiltonian $H_{s}$ of a charged (charge $e$ ) particle of mass $m$ in the considered system can be chosen in the form

$$
H_{s}=-\frac{\hbar^{2}}{2 m}\left[\left(\frac{\partial}{\partial x}+\frac{i e B}{c \hbar} y\right)^{2}+\frac{\partial^{2}}{\partial y^{2}}+\frac{\partial^{2}}{\partial z^{2}}\right]+\lambda \delta(y) .
$$

Here $B$ is the strength of the uniform magnetic field which is parallel to the $z$-axis, $\delta$ is the Dirac delta function, and $\lambda$ is a coupling constant of the system confinement

V. A. Geyler: Ogarev Univ. Mordovia, Dept. Math., Saransk 430000, Rūssia

I. V. Chudaev: Ogarev Univ. Mordovia, Dept. Math., Saransk 430000, Russia

ISSN 0232-2064 / \$2.50 (C) Heldermann Verlag Berlin 
potential $U(x, y, z)=\lambda \delta(y), \lambda<0$. In the momentum representation with respect to the momenta $p_{x}$ and $p_{z}$, the operator (1) has the form 2

$$
H_{s}=-\frac{\hbar^{2}}{2 m} \frac{\partial^{2}}{\partial y^{2}}+\frac{m \omega^{2}}{2}(y+q)^{2}+\lambda \delta(y)+\frac{1}{2 m} p_{z}^{2}
$$

where $\omega=\frac{|e B|}{c m}$ is the cyclotron frequency and $q=\frac{p_{x}}{\omega m}$ is the $y$-coordinate of the classical cyclotron orbit centre [18]. From (2) we obtain the following dispersion lows for the energy spectrum of $H_{3}$ :

$$
E_{n}\left(q, p_{z}\right)=E_{n}(q)+\frac{1}{2 m} p_{z}^{2}
$$

where $\left\{E_{n}(q): n \in \mathbb{N}\right\}$ is the set of all the eigenvalues of the perturbed harmonic oscillator

$$
H_{o s}=-\frac{\hbar^{2}}{2 m} \frac{\partial^{2}}{\partial y^{2}}+\frac{m \omega^{2}}{2} y^{2}+\lambda \delta(y-q) .
$$

It is important to note that choosing $\lambda>0$ in (1) we get a Hamiltonian of magnetosurface states in the presence of an in-plane magnetic field [7]. So in both cases $(\lambda>0$ and $\lambda<0$ ) we come to the spectral problem for an operator of the form

$$
H=-\frac{d^{2}}{d x^{2}}+V(x)+\lambda \delta(x-q)
$$

where the unperturbed operator $-\frac{d^{2}}{d x^{2}}+V(x)$ has a discrete spectrum. The principal interest in studying $H$ is investigating the $q$-dependence of the eigenvalues of the operator $H$.

Here are more examples of quantum mechanical systems for which the spectral analysis reduces to the study of an operators having the form (3).

1. Narrow conducting channel in an inversion layer or heterostructure subjected to a quantizing magnetic field perpendicular to the layer plane. The Hamiltonian of a charged particle in the system has the form

$$
H_{s}=-\frac{\hbar^{2}}{2 m}\left[\left(\frac{\partial}{\partial x}-\frac{i e B}{c h} y\right)^{2}+\frac{\partial^{2}}{\partial y^{2}}\right]+\lambda \delta(y)
$$

The recent progress in fabrication of two-dimensional electron systems with additional one-dimensional structures makes the possibility of experimental studying quantum transport in systems with Hamiltonian (4) (see, e.g., [25, 30, 31]).

2. Taking $\lambda>0$ in formula (4), we obtain a model of edge states in a twodimensional systern at the presence of a magnetic field $\{21,27]$, and the model of tunneling between quantum Hall systems [16].

3. Equation (3) describes a short-range impurity in a potential well. In this case $V(x)$ is the confining potential of the wcll and $\lambda$ is the strength of the impurity potential ( $\lambda<0$ for attractive impurities and $\lambda>0$ for repulsive ones). The considered model 
was employed, e.g., in $[10,19,22,24]$. Besides, taking $\lambda>0$ we obtain the Hamiltonian of a charged particle tunneling through a moving barrier $[6,29]$.

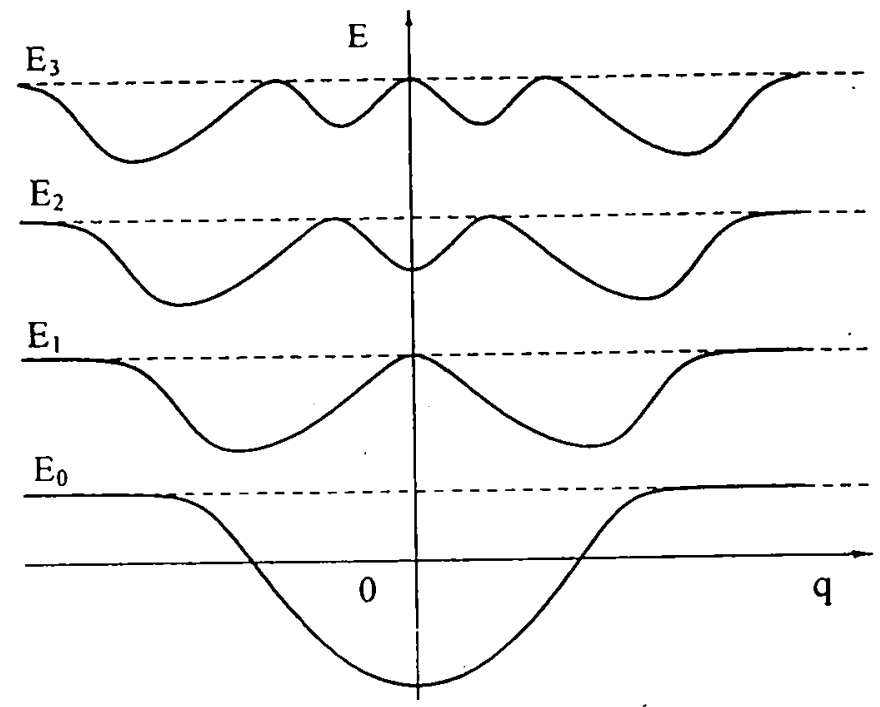

Figure 1: The plot of the eigenvalues $E_{n}(q)$ as functions of $q$ in the case of $\lambda<0$

In the papers cited, the $q$-dependence of the spectrum of $H$ has been studied by mumerical methods. In the case of a harmonic oscillator potential $V\left(V(x)=\frac{x^{2}}{4}\right)$ the typical plot of the eigenvalues $E_{n}(q)$ as functions of $q$ is presented on Figures $1-3$.

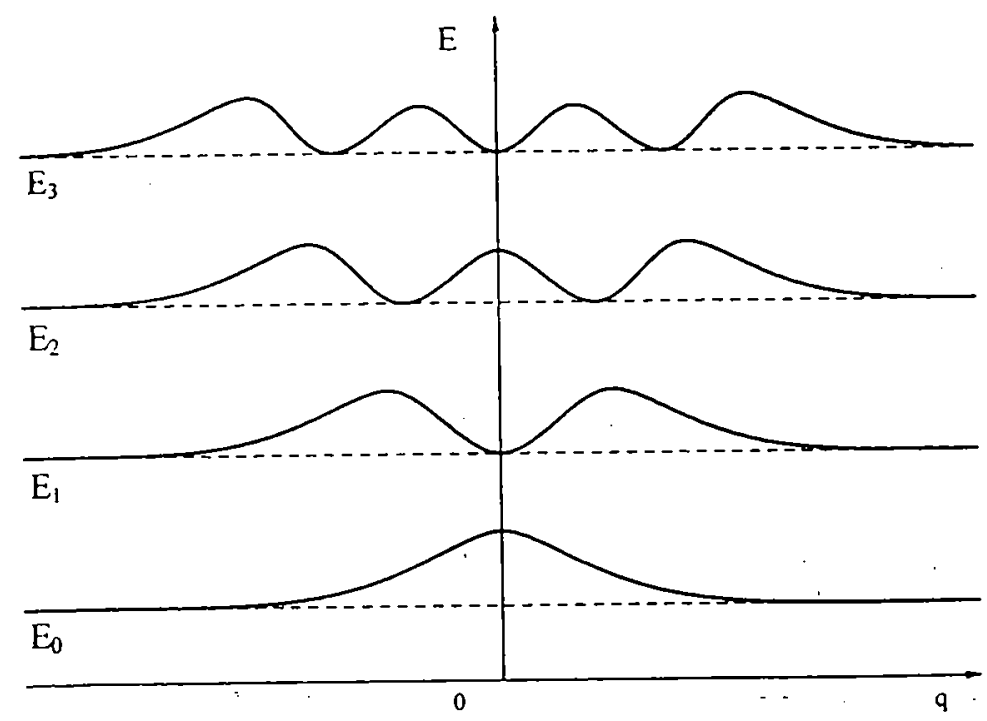

Figure 2: The plot of the eigenvalues $E_{n}(q)$ as functions of $q$ in the case of $\lambda>0$ 
They present the case of $\lambda<0, \lambda>0$ and $\lambda=0$, respectively. On these figures; $\epsilon_{n}$ are the levels of the harmonic oscillator: $\epsilon_{n}=\frac{n+1}{2}$. The peculiarities of the plots are used for explanation of series of experimental data (see, e.c., $[8,14,33]$ ). As far as we know, there is no strict mathematical analysis of these peculiarities in the general case. For the case of a harmonic oscillator potential $V(x)$ such an analysis is presented in [13]; detailed proofs are contained in [11]. The proofs of main results of the mentioned paper are based on specific properties of the Green function of the harmonic oscillator and are not extended to more general potentials $V(x)$. The aim of the present paper is to present strict mathematical results explaining the above-mentioned peculiaritics in the case of a sufficiently general form of the potential $V(x)$ in equation (3). We use an approach based on operator extension theory $[2,28]$. In this approach, the Krein resolvent formula for selfadjoint extensions of symmetric operator plays the crucial role [17]. The possibility to use the Green function version of this formula for operators of the form (3) follows from results of $[1,12]$.

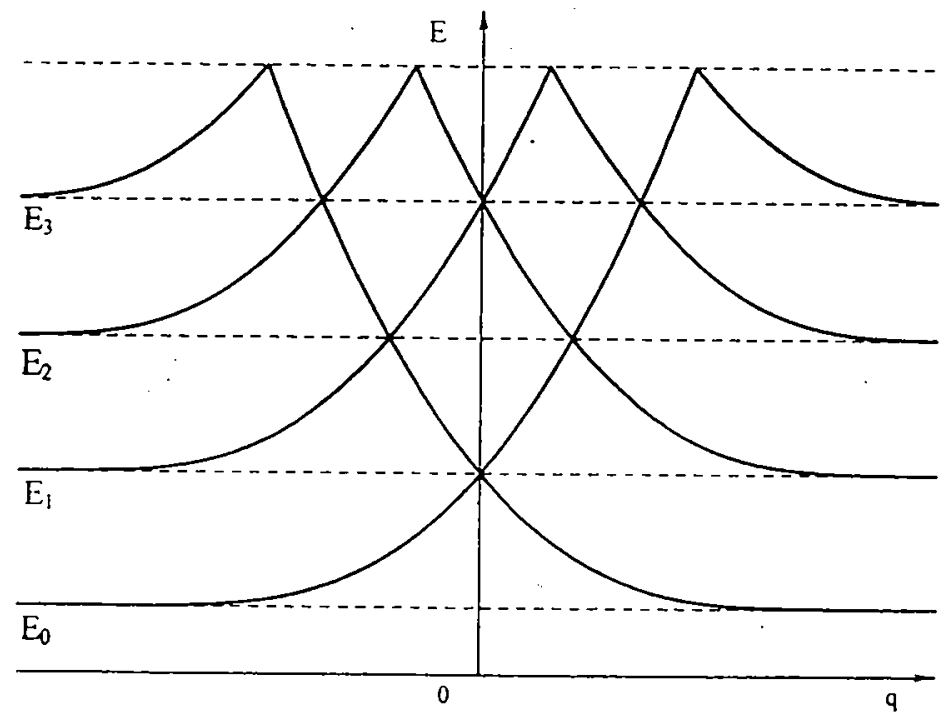

Figure 3: The plot of the eigenvalues $E_{n}(q)$ as functions of $q$ in the case of $\lambda=0$

\section{Preliminaries}

In this section we introduce basic notations and state some auxiliary results (we refer to $[9,26]$ for details). During the paper, $H_{0}$ denotes a self-adjoint operator in the space $L^{2}(a, b) \quad(-\infty \leq a<b \leq+\infty)$ generated by a formally self-adjoint differential expression $\tau$ of order $2 \nu$ :

$$
\tau(f)=(-1)^{\nu}\left(p_{0} f^{(\nu)}\right)^{(\nu)}+(-1)^{(\nu-1)}\left(p_{1} f^{(\nu-1)}\right)^{(\nu-1)}+\ldots+p_{\nu} f .
$$

To avoid some pathologies we suppose that the following conditions are valid: 
(L1) The derivatives (in sense of distributions) of the functions $p_{k} \quad(0 \leq k \leq \nu-1)$ up to order $\nu-k-1$ are locally absolutely continuous functions on $(a, b)$.

(L2) $p_{0}(x) \neq 0$ for all $x \in(a, b)$.

(L3) $p_{\nu} \in L_{l o c}^{2}(a, b)$.

In particular, under these conditions we have

$$
C_{0}^{\infty}(a, b) \subset \mathcal{D}\left(H_{0}\right) \subset C(a, b)
$$

where $\mathcal{D}\left(H_{0}\right)$ is the domain of $H_{0}$.

As usually, $\sigma\left(H_{0}\right)$ denotes the spectrum of the operator $H_{0}, \rho\left(H_{0}\right)=\mathbb{C} \backslash \sigma\left(H_{0}\right)$, and $R_{0}(\zeta)$ denotes the resolvent of the operator $H_{0}: R_{0}(\zeta)=\left(H_{0}-\zeta\right)^{-1}$. By $I$ we denote the interval $(a, b)$ with added regular endpoints. The operator $R_{0}(\zeta)$ has an integral kernel $G_{0}(x, y ; \zeta)$ (the Green function of $H_{0}$ ), this function satisfies the following conditions:

(G1) $\int_{a}^{b}\left|G_{0}(x, y ; \zeta)\right|^{2} d y<\infty$ for all $x \in I$ and $\zeta \in \rho\left(H_{0}\right)$.

(G2) If $m, n \in \mathbb{N}$ and $m+n \leq 2 \nu-1$, then all the functions $\partial_{x}^{m} \partial_{y}^{n} G_{0}(x, y ; \zeta)$ are continuous in the region $I \times I \times \rho\left(H_{0}\right)$ and holomorphic in $\zeta \in \rho\left(H_{0}\right)$ for any fixed $x$ and $y$.

Finally, the following supposition will be always assumed:

(S) The spectrum of the operator $H_{0}$ is discrete and bounded from below: $\sigma\left(H_{0}\right)=$ $\left\{\lambda_{n}: n \in \mathbb{N}\right\}$ where $\lambda_{0}<\lambda_{1}<\ldots<\lambda_{n}<\ldots$.

We shall denote by $\kappa(n)$ the multiplicity of the eigenvalue $\lambda_{n}$; it is well-known that $\kappa(n) \leq 2 \nu$. For every $n$ we fix an orthonormal system $\Phi_{n, k}(k=1, \ldots, \kappa(n))$ of eigenfunctions of the operator $H_{0}$ corresponding to the eigenvalue $\lambda_{n}$. Then

$$
G_{0}(x, y ; \zeta)=\sum_{n=0}^{\infty} \sum_{k=1}^{\kappa(n)}\left(\lambda_{n}-\zeta\right)^{-1} \Phi_{n, k}(x) \overline{\Phi_{n, k}(y)}
$$

and by virtue of the Mercer theorem the series

$$
\sum_{n=0}^{\infty} \sum_{k=1}^{\kappa(n)}\left|\left(\lambda_{n}-\zeta\right)^{-1} \Phi_{n, k}(x) \overline{\Phi_{n, k}(y)}\right|
$$

converges locally uniformly on $I \times I \times \rho\left(H_{0}\right)$.

Let $q \in(a, b)$ and $-\infty<\mu<+\infty, \mu \neq 0$. We shall consider the self-adjoint operator $H . \equiv H(q, \mu)$ in the space $L^{2}(a, b)$ formally defined by the expression

$$
H(q, \mu)=H_{0}+\mu \delta(x-q) .
$$


To attach a rigorous mathematical meaning to the expression (7) we shall use the Krein resolvent formula [17]. According to that formula $H$ is defined by its resolvent $R(\zeta)$ which has the integral kernel

$$
G(x, y ; \zeta)=G_{0}(x, y ; \zeta)-\left[Q(\zeta)+\mu^{-1}\right]^{-1} G_{0}(x, q ; \zeta) G_{0}(q, y ; \zeta)
$$

where $Q(\zeta)$ is the so-called Krein function. Using equation (2) and property (G1) we can show that $Q(\zeta)$ has the form $Q(\zeta) \equiv Q(\zeta, q)=G_{0}(q, q ; \zeta)$ (see [12] for details). Hence

$$
Q(\zeta)=\sum_{n=0}^{\infty} \sum_{k=1}^{\kappa(n)}\left(\lambda_{n}-\zeta\right)^{-1}\left|\Phi_{n, k}(q)\right|^{2}
$$

Formula (8) allows one to consider the operator $H(q, \mu)$ at $\mu=+\infty$; it can be shown that in this case $H(q, \mu)$ is the direct sum of two opcrators on the intervals $(a, q)$ and $(q, b)$, respectively, with Dirichlet boundary conditions at the point $q$.

We start with some auxiliary assertions.

Lemma 1. Let $P_{0}$ and $P_{1}$ are orthoprojections in a Hilbert space, and let $\alpha \in \mathbb{C}$. If $P_{0}+\alpha P_{1}$ is an orthoprojection, then $\alpha$ equals one of the numbers $0,1,-1$.

Lemma 2. For every $x_{0} \in(a, b)$ the relation $\Phi_{n, k}\left(x_{0}\right) \neq 0$ is valid for infinitely many values of the index $n$.

Proof. Otherwise, we obtain from (5) that there exists a function $h \in C(a, b)$ which is a finite linear combination of functions $\Phi_{n, k}$ and obeys $\varphi\left(x_{0}\right)=\langle h \mid \varphi\rangle$ for all $\varphi \in C_{0}^{\infty}(a, b)$. Obviously, this is impossible

Lemma 3. For a fixed $q \in(a, b)$ the following statements are true:

(i) $\zeta \mapsto Q(\zeta, q)$ is a meromorphic function of $\zeta \in \mathbb{C}$ with infinitely many simple poles. The poles of this function are exactly those points $\lambda_{n}$ for which there exists $k \in\{1, \ldots, \kappa(n)\}$ such that $\Phi_{n, k}(q) \neq 0$.

(ii) $\frac{\partial Q(\zeta, q)}{\partial \zeta}>0$ if $\zeta \in \rho\left(H_{0}\right) \cap \mathbb{R}$.

(iii) For real $E$ the function $E \mapsto Q(E, q)$ increases from $-\infty$ to $+\infty$ as $E$ varies between any two neighbouring poles.

(iv) For every $\zeta \in \rho\left(H_{0}\right)$ the function $x \mapsto G_{0}(x, q ; \zeta)$ do not vanish identically on the interval $(a, b)$. $(a, b)$.

(v) $Q(\zeta, q) \rightarrow 0$ as $\Re \zeta \rightarrow-\infty$ locally uniformly uith respect to $q$ on the interval

Proof. It follows immediately from Lemma 2 and formulae (6) and (9)

The following theorem establishes a relation between formulae (7) and (8); the proof of the theorem can be found in [1]. Before to formulate the theorem, we remind that the symbol $\mathcal{Q}(H)$ denotes as usually the form domain of $H$. 
Theorem 1. Let $\mu \neq+\infty$. Then the following statements are true:

(i) $\mathcal{Q}\left(H_{0}\right)=\mathcal{Q}(H) \subset C(a, b)$.

(ii) If $\psi \in \mathcal{Q}(H)$, then $h(\psi)=h_{0}(\psi)+\mu|\psi(q)|^{2}$ where $h$ and $h_{0}$ are the quadratic forms associated with the operators $H$ and $H_{0}$; respectively.

(iii) The quadratic form $|\psi(q)|^{2} \quad\left(\psi \in \mathcal{Q}\left(H_{0}\right)\right)$ is infinitesimal small with respect to the form $h_{0}$.

\section{Spectral properties of the operator $H(q, \mu)$ for fixed $q$}

Fix a point $q \in \mathbb{R}$, and let $\lambda_{n_{0}}<\lambda_{n_{1}}<\ldots<\lambda_{n_{k}}<\ldots$ be the increasing sequence of all the poles of the function $\zeta \mapsto Q(\zeta, q)$ (by virtue of Lemma 3 this sequence is infinite). We shall assume further that if a point $\zeta=\lambda_{n}$ does not belong to the sequence, then the function $Q(\zeta, q)$ is equal to its continuous extension to the point. In the spectral analysis of the operator $H(q, \mu)$, the key part is played by the following equation with respect to the spectral parameter $\zeta$ (known as dispersion equation):

$$
Q(\zeta, q)+\mu^{-1}=0
$$

For any $\zeta \neq \lambda_{n_{k}}$ the function $G_{0}(\cdot, q ; \zeta)$ does not vanish identically (Lemmas 2 and $3)$. Hence by virtue of formula (8) every $\zeta$ satisfying equation (10) is a pole of the resolvent $R(\zeta)$ and therefore belongs to the spectrum $\sigma(H)$. Thus equation (10) has only real solutions. It follows from Lemma 3 that for every $\mu$ this equation has exactly one solution in each interval $\left(\lambda_{n_{0}}, \lambda_{n_{1}}\right),\left(\lambda_{n_{1}}, \lambda_{n_{2}}\right), \ldots$. We arrange these solutions in an increasing sequence $\mathcal{E}_{1}(q)<\mathcal{E}_{2}(q)<\ldots$. If $\mu>0$, equation (10) has no other solutions; otherwise it has an additional solution $\mathcal{E}_{0}(q)$ lying in the interval $\left(-\infty, \lambda_{n_{0}}\right)$.

Theorem 2. The spectrum of the operator $H(q, \mu)$ is a discrete one and consists of four non-intersecting parts $\sigma_{k}(k=1, \ldots, 4)$ which are described as follows.

(i) The part $\sigma_{1}$ consists of all the solutions $\mathcal{E}_{j}(q)$ of equation (10) which are differcnt from the eigenvalues $\lambda_{n}$ of the operator $H_{0}$. Each solution $\mathcal{E}_{j}(q)$ is a simple eigenvalue of the operator $H(q, \mu)$, the corresponding normalized eigenfunction has the form

$$
\widehat{\Phi}_{j}(x)=G_{0}\left(x, q ; \mathcal{E}_{j}(q)\right)\left[\frac{\partial Q}{\partial E}\left(\mathcal{E}_{j}(q), q\right)\right]^{-\frac{1}{2}}
$$

(ii) The part $\sigma_{2}$ consists of all the eigenvalues $\lambda_{m}$ of the operator $H_{0}$ satisfying equation (10) and such that $\Phi_{m, k}(q)=0$ for all $k=1, \ldots \kappa(m)$. Each point of $\sigma_{2}$ is an eigenvalue of the operator $H(q, \mu)$ of multiplicity $\kappa(m)+1$; an orthonormal bascs of the corresponding eigensubspace is spunned by the functions $\Phi_{m, k}$ and the function

$$
\Psi_{m}(x)=G_{0}\left(x, q ; \lambda_{m}\right)\left[\frac{\partial Q}{\partial E}\left(\lambda_{m}, q\right)\right]^{-\frac{1}{2}} .
$$

(iii) The part $\sigma_{3}$ consists of all the eigenvalues $\lambda_{m}$ of the operator $H_{0}$ which do not satisfy equation (10) and such that $\Phi_{m, k}(q)=0$ for all $k=1, \ldots, \kappa(m)$. Each point of 
$\sigma_{3}$ is an eigenvalue of the operator $H(q, \mu)$ of multiplicity $\kappa(m)$; an orthonormal bases of the corresponding eigensubspace is spanned by the functions $\Phi_{m, k}$.

(iv) The part $\sigma_{4}$ consists of all the eigenvalues $\lambda_{m}$ of the operator $H_{0}$ for which $\kappa(m) \geq 2$ and which are poles of the function $\zeta \mapsto Q(\zeta, q)$ (i.e., for which $\Phi_{m, k}(q) \neq 0$ at least for one $k)$. Each point of $\sigma_{4}$ is an eigenvalue of the operator $H(q, \mu)$ of multiplicity $\kappa(m)-1$; the corresponding eigensubspace is the orthogonal complement of the function

$$
\widehat{F}_{m}(x)=\overline{\Phi_{m, 1}(q)} \Phi_{m, 1}(x)+\ldots,+\overline{\Phi_{m, \kappa(m)}(q)} \Phi_{m, \kappa(m)}(x)
$$

in the eigensubspace of the operator $H_{0}$ associated with $\lambda_{m}$.

Proof. It is convenient now to rewrite the Krein formula (8) in the operator form

$$
R(\zeta)=R_{0}(\zeta)-\left[Q(\zeta, q)+\mu^{-\mathrm{i}}\right]^{-1} g_{q}(\zeta)\left\langle g_{q}(\zeta) \mid \cdot\right\rangle
$$

where $g_{q}(\zeta)=G_{0}(\cdot, q ; \zeta)$. Let us introduce the notation

$$
A(\zeta)=\left[Q(\zeta, q)+\mu^{-1}\right]^{-1} g_{q}(\zeta)\left\langle g_{q}(\zeta) \mid \cdot\right\rangle
$$

Further, we shall use the following well-known assertion: the orthoprojector $P$ on the eigensubspace of a selfajoint operator $T$ corresponding to an isolated point $E_{0}$ of its spectrum has the form

$$
P=-\operatorname{Res}\left[(T-\zeta)^{-1} ; E_{0}\right] \text {. }
$$

If $\mathcal{E}_{j}(q) \neq \lambda_{n}$ for all $n$, then from (11) it follows immediately that $\mathcal{E}_{j}(q)$ is a pole of the resolvent $R(\zeta)$. In this case equation (12) determines the orthoprojector $P$ on the subspace spanned by the vector $g_{q}\left(\mathcal{E}_{j}(q)\right)$. An elementary calculation of the residue leads to the expression $P=\hat{g}_{q}\left(\mathcal{E}_{j}(q)\right)\left\langle\hat{g}_{q}\left(\mathcal{E}_{j}(q)\right) \mid \cdot\right\rangle$ where

$$
\hat{g}_{q}\left(\mathcal{E}_{j}(q)\right)=g_{q}\left(\mathcal{E}_{j}(q)\right)\left[\frac{\partial Q}{\partial E}\left(\mathcal{E}_{j}(q), q\right)\right]^{-\frac{1}{2}}
$$

Let now $\mathcal{E}_{j}(q)=\lambda_{m}$ where $\lambda_{m}$ is not a pole of the function $\zeta \mapsto Q(\zeta, q)$, i.e. let $\Phi_{m, k}(q)=0$ for all $k=1, \ldots, \kappa(m)$. Then in a neighbourhood of the point $\lambda_{m}$ we have

$$
Q(\zeta, q)+\mu^{-1}=\alpha_{1}\left(\zeta-\lambda_{m}\right)+\alpha_{2}\left(\zeta-\lambda_{m}\right)^{2}+\ldots
$$

where $\alpha_{1}=\frac{\partial Q}{\partial \zeta}\left(\lambda_{m}, q\right)>0$. Thus $\operatorname{Res}\left[A(\zeta) ; \lambda_{m}\right]=\alpha P_{1}$ where $\alpha>0$ and $P_{1}$ is the orthoprojector on the subspace spanned by the vector $g_{q}\left(\lambda_{m}\right)$. Hence $-\operatorname{Res}\left[R(\zeta) ; \lambda_{m}\right]=$ $P_{0}+\alpha P_{1}$, where $P_{0}=-\operatorname{Res}\left[R_{0}(\zeta) ; \lambda_{m}\right] ; P_{0}$ is the orthoprojector on the subspace spanned by the functions $\Phi_{m, k}(k=1, \ldots, \kappa(m))$. Using Lemma 1 , we obtain that $\alpha=1$. Therefore

$$
P \equiv-\operatorname{Res}\left[R(\zeta) ; \lambda_{m}\right]=P_{0}+P_{1}
$$

It follows from equation (13) that $P$ is the orthoprojector on the subspace spanned by the functions $\Phi_{m, k}$ and the function $\Psi_{m}$ from item (ii) of the theorem.

Let us suppose now that $\lambda_{m}$ is not a pole of the function $\zeta \mapsto Q(\zeta, q)$ and is not a solution of equation (10), i.e. it is not equal to any $\mathcal{E}_{j}(q)$. Then $\operatorname{Res}\left[A(\zeta) ; \lambda_{m}\right]=0$, 
therefore $-\operatorname{Res}\left[R(\zeta) ; \lambda_{m}\right]=-\operatorname{Res}\left[R_{0}(\zeta) ; \lambda_{m}\right]$. Thus $\lambda_{m}$ is an eigenvalue of the operator $H$ associated with the eigensubspace spanned by the vectors $\Phi_{m, k}$.

Finally, let $\lambda_{m}$ be a pole of the function $\zeta \mapsto Q(\zeta, q)$, i.e. $\Phi_{m, k}(q) \neq 0$ at least for one $k \in\{1, \ldots, \kappa(m)\}$. Then $\lambda_{m}$ is a pole of the function $\zeta \mapsto g_{q}(\zeta)$; the corresponding residue we denote by $\hat{F}_{m}$. Hence $\operatorname{Res}\left[A(\zeta) ; \lambda_{m}\right]=\beta P_{1}$ where $\beta \neq 0$ and $P_{1}$ is the orthoprojector on the subspace spanned by the function $\widehat{F}_{m}(x)$. Therefore $P \equiv-\operatorname{Res}\left[R(\zeta) ; \lambda_{m}\right]=P_{0}+\beta P_{1}$. Because $P$ is an orthoprojector and $P \neq P_{0}$, then by virtue of Lemma $1, \beta= \pm 1$. Since the range of $P_{1}$ is contained in the range of $P_{0}$, then $\beta=-1$. Since $P_{1}$ is a one-dimensional projector, $\operatorname{dim} \operatorname{Rang} P=\operatorname{dim} \operatorname{Rang} P_{0}-1$. The theorem is proved

\section{The dispersion laws $E_{n}(q)$}

Our next aim is to analyse the $q$-dependence of the eigenvalues $\mathcal{E}_{j}(q)$. Since the set $\left\{\lambda_{n_{k}}\right\}$ of the poles of the function $\zeta \mapsto Q(\zeta, q)$ depends on $q$, the use of the eigenvalues $\mathcal{E}_{j}(q)$ is not relevant to the study of their $q$-dependence properties. In this connection we give another parametrization of the eigenvalues of $H$. As a preliminary step we introduce the notations

$$
\begin{aligned}
\lambda_{-1} & =-\infty \\
X_{-1} & =\mathbb{R} \\
X_{n} & =\left\{q \in \mathbb{R}: \Phi_{n, j}(q) \neq 0 \text { for all } j=1, \ldots, \kappa(n)\right\} \quad(n \geq 0) .
\end{aligned}
$$

Since each function $\Phi_{n, j}$ is a solution of a linear differential equation, the sets $\mathbb{R} \backslash$ $X_{n}$ are discrete. The function $E \mapsto Q(E, q)$ is continuously differentiable on each set $\left(\lambda_{k-1}, \lambda_{k}\right) \times\left(X_{k-1} \cap X_{k}\right)$ and real-analytic on the interval $\left(\lambda_{k-1}, \lambda_{k}\right)$ as a function of $E$ for any fixed $q \in X_{k-1} \cap X_{k}$. As is shown above, equation (10) for any $q \in X_{k-1} \cap X_{k}$ has just one solution belonging to the interval $\left(\lambda_{k-1}, \lambda_{k}\right)$; we denote this solution by $E_{k}(q)$. Recall that the index $k$ runs from 1 if $\mu>0$ and from 0 if $\mu<0$. Because $\frac{\partial Q}{\partial E} \neq 0$ on the set $\left(\lambda_{k-1}, \lambda_{k}\right) \times\left(X_{k-1} \cap X_{k}\right)$, the implicit function theorem implies that $E_{k}(q)$ is a continuously differentiable function on the set $X_{k-1} \cap X_{k}$.

Proposition 1. Each function $E_{k}(q)$ has a continuous extension on the whole real line $\mathbb{R}$.

Proof. We fix $s \in \mathbb{R} \backslash X_{k-1} \cap X_{k}$ and choose a sequence $\left(q_{n}\right) \subset X_{k-1} \cap X_{k}$, with $q_{n} \rightarrow s$. From Lemma 3 it is easy to show that the sequence $E_{k}\left(q_{n}\right)$ is bounded, hence, we can extract a subsequence $E_{k}\left(q_{n_{m}}\right)$ converging to a limit which we denote by $\hat{E}$. To complete the proof of the statement we need two lemmas.

Lemma 4. The following assertions are true:

(i) The number $\widehat{E}$ is not a pole of the function $\zeta \mapsto Q(\zeta, s)$.

(ii) If $\lambda_{k-1}<\widehat{E}<\lambda_{k}$, then $\zeta=\widehat{E}$ is a solution of the equation $Q(\zeta, s)+$ $\mu^{-1}=0$ (see (10)), and there exists no other solutions of the equation on the interval $\left(\lambda_{k-1}, \lambda_{k}\right)$. 
(iii) If $\widehat{E}=\lambda_{k-1}$, then $\lim _{E \rightarrow \widehat{E}}\left[Q(E, s)+\mu^{-1}\right] \geq 0$.

(iv) If $\widehat{E}=\lambda_{k}$, then $\lim _{E \rightarrow \widehat{E}}\left[Q(E, s)+\mu^{-1}\right] \leq 0$.

Proof. Assertion (i): Let $f(\zeta)=\left(\zeta-\lambda_{k-1}\right)\left(\zeta-\lambda_{k}\right)$; we consider the function

$$
\widehat{Q}_{\mu}(\zeta, q)=\left[Q(\zeta, q)+\mu^{-1}\right] f(\zeta),
$$

which is continuous on the set $\left(\lambda_{k-2}, \lambda_{k+1}\right) \times \mathbb{R}$. Since $\hat{Q}_{\mu}\left(E_{k}\left(q_{n_{m}}\right), q_{n_{m}}\right)=0$, then by passing to the limit $m \rightarrow \infty$ we obtain

$$
\widehat{Q}_{\mu}(\widehat{E}, s)=0 .
$$

If $\widehat{E}$ is a pole of the function $\zeta \mapsto Q(\zeta, s)$, then $\widehat{Q}_{\mu}(\hat{E}, s) \neq 0$ by virtue of Formula (14), what contradicts to the equation (15).

Assertion (ii): Taking into account Lemma 3/(ii), we obtain the statement of the item by passing to the limit in the equality $Q\left(E_{k}\left(q_{n_{m}}\right), q_{n_{m}}\right)+\mu^{-1}=0$.

Assertion (iii): By virtue of item (i) the function $\zeta \rightarrow Q(\zeta, s)$ is continuous in a

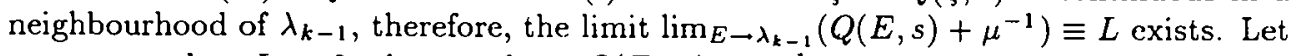
us assume that $L<0$, then we have $Q\left(E_{0}, s\right)+\mu^{-1}<0$ for some $E_{0} \in\left(\lambda_{k-1}, \lambda_{k}\right)$. Hence for some $m$ the inequalities $Q\left(E_{0}, s\right)+\mu^{-1}<0$ and $E_{k}\left(q_{n_{m}}\right)<E_{0}$ arc satisfied simultaneously. Since $Q(E, q)$ increases on the interval $\left(\lambda_{k-1}, \lambda_{k}\right)$ as function of $E$, we obtain the contradiction $0<Q\left(E_{k}\left(q_{n_{m}}\right), q_{n_{m}}\right)+\mu^{-1}<Q\left(E_{0}, q_{n_{m}}\right)+\mu^{-1}<0$.

Assertion (iv): The proof of this item is similar to that of item (iii)

Lemma 5. For any sequence $\left(s_{n}\right) \subset X_{k-1} \cap X_{k}$ converging to $s$ the relation $E_{k}\left(s_{n}\right) \rightarrow \widehat{E}$ takes place.

Proof. Suppose that for some sequence $\left(s_{n}\right) \subset X_{k-1} \cap X_{k}$ we have $s_{n} \rightarrow s$, but $E_{k}\left(s_{n}\right) \nrightarrow \hat{E}$. Then we can choose a subsequence $\left(s_{n_{k}}\right)$ such that $E_{k}\left(s_{n_{m}}\right) \rightarrow E^{*}$, and $E^{*} \neq \widehat{E}$. Suppose that $E^{*}>\widehat{E}$.

Taking into account Lemma $4 /($ ii) and the fact that $Q$ is strictly increasing of $E$, we show that $\widehat{E}=\lambda_{k-1}$ or $E^{*}=\lambda_{k}$. In both cases we have

$$
\lim _{E \rightarrow \widehat{E}}\left[Q(E, s)+\mu^{-1}\right] \geq 0 \quad \text { and } \quad \lim _{E \rightarrow E^{\bullet}}\left[Q(E, s)+\mu^{-1}\right] \leq 0 .
$$

Let us take some numbers $E_{1}$ and $E_{2}$ so that $\widehat{E}<E_{1}<E_{2}<E^{*}$. Then from (16) with regard to the strict monotonicity of $Q$ as function of $E$ we have

$$
0 \leq Q\left(E_{1}, s\right)+\mu^{-1}<Q\left(E_{2}, s\right)+\mu^{-1} \leq 0 .
$$

This is a contradiction. Taking into account Lemmas 4 and 5 , we complete the proof of the Proposition 1

The theorem below is the main result of this section. 
Theorem 3. For each fixed $0 \neq \mu \in(-\infty,+\infty]$ there is a sequence $\left(E_{n}(q)\right)$ of continuous functions of $q \in \mathbb{R}$ such that the following properties are satisfied:

(i) $\lambda_{n-1} \leq E_{n}(q) \leq \lambda_{n}$ for all $n$.

(ii) $E_{n}(q) \leq E_{n+1}(q)$ for all admissible values of $n$.

(iii) For each $q$ the set consisting of all $E_{n}(q)$ and all the numbers $\lambda_{m}$ with $\kappa(m) \geq 2$ is the complete collection of all the eigenvalues of the operator $H(q, \mu)$.

(iv) If $\lambda_{n-1}<E_{n}(q)<\lambda_{n}$, then $E_{n}(q)$ is the unique solution of the dispersion equation (10).

(v) If $\lambda_{n}$ is a pole of the function $\zeta \mapsto Q(\zeta, q)$ (i.e. if $\Phi_{n, k}(q) \neq 0$ at least for one $k)$, then $\lambda_{n-1}<E_{n}(q)<\lambda_{n}$.

(vi) Provided that $\lambda_{n}$ is not a pole of the function $\zeta \mapsto Q(\zeta, q)$ (i.e. $\Phi_{n, k}(q)=0$ for all $k$ ), we have the following:

(a) If $Q\left(\lambda_{n}, q\right)+\mu^{-1}<0$, then $E_{n}(q)=\lambda_{n}<E_{n+1}(q)$.

(b) If $Q\left(\lambda_{n}, q\right)+\mu^{-1}>0$, then $E_{n}(q)<\lambda_{n}=E_{n+1}(q)$.

(c) If $Q\left(\lambda_{n}, q\right)+\mu^{-1}=0$, then $E_{n}(q)=\lambda_{n}=E_{n+1}(q)$.

Definition. The points $q$ for which condition (iv) of Theorem 3 is satisfied we shall call the non-singular points of the function $E_{n}(q)$. If condition (vi)/(a), (vi)/(b) or (vi)/(c) is satisfied, then $q$ will be called singular point of kind (a), (b) or (c), respectively.

Proof of Theorem 3. By virtue of Proposition 1 each function $E_{n}(q)$ can be extended by continuity on the whole real line $\mathbb{R}$; the extension we denote by $E_{n}(q)$ again. Properties (i) and (ii) of the function $E_{n}(q)$ are obvious, property (iii) follows from Theorem 2 and properties (iv) and (vi). Properties (iv) and (v) follow immediately from the definition of the functions $E_{n}(q)$ and Lemma 4 . It remains to prove property (vi).

Let $\lambda_{n}$ be not a pole of the function $\zeta \mapsto Q(\zeta, q)$, and let $Q\left(\lambda_{n}, q\right)+\mu^{-1}<0$. For any positive integer $m$ we choose a number $E_{m}$ such that $\lambda_{n}-\frac{1}{m}<E_{m}<\lambda_{n}$; then $Q\left(E_{m}, q\right)+\mu^{-1}<0$. Further, we choose points $q_{m} \in \mathbb{R}$ for which $\lambda_{n-1}$ and $\lambda_{n}$ are not the poles of the function $\zeta \mapsto Q\left(\zeta, q_{m}\right)$ (that is $q_{m} \in X_{m-1} \cap X_{m}$ ), and such that $\left|q-q_{m}\right|<\frac{1}{m}$ and $Q\left(E_{m}, q_{m}\right)+\mu^{-1}<0$. Then $E_{n}\left(q_{m}\right)$ is a solution of the equation $Q\left(E, q_{m}\right)+\mu^{-1}=0$ laying in the interval $\left(\lambda_{n-1}, \lambda_{n}\right)$. Since $Q$ is a strictly monotone function of $E$ in this interval, the inequalities $E_{m}<E_{n}\left(q_{m}\right)<\lambda_{n}$ take place for all $m$. Thus $E_{n}\left(q_{m}\right) \rightarrow \lambda_{n}$ and $q_{m} \rightarrow q$ as $m \rightarrow \infty$; therefore $\lambda_{n}=E_{n}(q)$ by the definition of the function $E_{n}(q)$. According to Lemma $4 /(\mathrm{iii}), Q\left(\lambda_{n}, q\right)+\mu^{-1} \geq 0$ if $\lambda_{n}=E_{n+1}(q)$; therefore $\lambda_{n}<E_{n+1}(q)$. Thus item (vi)/(a) is proved. The proofs of items (vi)/(b) and (vi)/(c) are similar 


\section{Properties of the dispersion lows $E_{n}(q)$ for Schrödinger operators}

Here we consider the most important class of operators $H_{0}$, namely Schrödinger operators on the real line $\mathbb{R}$. Throughout the section, $H_{0}$ denotes the operator in $L^{2}(\mathbb{R})$ given by the differential expression

$$
\tau=-\frac{d^{2}}{d x^{2}}+V(x)
$$

where the potential $V$ belongs to the space $L_{\text {loc }}^{2}(\mathbb{R})$ and is bounded from below. In the considered case the expression (17) defines an essentially selfadjoint operator on $C_{0}^{\infty}(\mathbb{R})$.

We need some additional restrictions on the potential $V$. First of all we shall assume that the condition

(V1) $\lim _{x \rightarrow \pm \infty} V(x)=+\infty$

is fulfilled. Under this condition the operator $H_{0}$ has a simple discrete spectrum, and each eigenfunction $\Phi_{m} \equiv \Phi_{m, 1}$ has strictly $m$ zeros on the real line. In addition, we can suppose that all the functions $\Phi_{m}$ are real-valued ones.

In the case of the Schrödinger operator, the following representation of the Green function $G_{0}$ is convenient. Let $\zeta_{0} \in \rho\left(H_{0}\right)$. Then the equation $H_{0} \Psi=\zeta_{0} \Psi$ has up to a multiplicative constant only one non-zero square integrable solution $\Psi_{+}\left(x ; \zeta_{0}\right)$ and $\Psi_{-}\left(x ; \zeta_{0}\right)$ on the half-lines $(0,+\infty)$ and $(-\infty, 0)$, respectively. Let

$$
\omega\left(\zeta_{0}\right)=W\left(\Psi_{+}\left(x ; \zeta_{0}\right), \Psi_{-}\left(x ; \zeta_{0}\right)\right)
$$

be the Wronskian of these solutions. Then

$$
G_{0}\left(x, y ; \zeta_{0}\right)=\frac{\Psi_{+}\left(\max (x, y) ; \zeta_{0}\right) \Psi_{-}\left(\min (x, y) ; \zeta_{0}\right)}{\omega\left(\zeta_{0}\right)}
$$

(see [32]). In a neighbourhood of the point $\zeta_{0}$ the functions $\Psi_{+}(x ; \zeta)$ and $\Psi_{-}(x ; \zeta)$ can be chosen to be analytical ones of $\zeta$. In addition, the function $\omega(\zeta)$ has only simple zeros, and these zeros coincide with the eigenvalues $\lambda_{n}$ of the operator $H_{0}$. From (18) we obtain

$$
Q(\zeta, q)=\frac{\Psi_{+}(q ; \zeta) \Psi_{-}(q ; \zeta)}{\omega(\zeta)}
$$

Let $\zeta \in \mathbb{R}$ and $\zeta<\lambda_{0}$. Then the functions $\Psi_{+}(x ; \zeta)$ and $\Psi_{-}(x ; \zeta)$ can be chosen to be strictly positive for all $x \in \mathbb{R}$. For the same values of $\zeta$, the Green function $G_{0}$ is the integral kernel of a positive operator, therefore

$$
Q(\zeta, q)=G_{0}(q, q ; \zeta) \geq 0
$$

for all $q \in \mathbb{R}$. On the other hand, by virtue of Lemma $3, \frac{\partial Q}{\partial \zeta}>0$ and $\lim _{\zeta \rightarrow-\infty} Q(\zeta, q)=$ 0 , hence for all $q \in \mathbb{R}$ and for the same values of $\zeta$, we have

$$
Q(\zeta, q)=G_{0}(q, q ; \zeta)>0
$$


In particular, we have

$$
\omega(\zeta)>0
$$

The case of even potentials $V(x)$ is of principal interest. In this case the functions $\Phi_{2 m}$ are even and $\Phi_{2 m+1}$ are odd. Besides, we can assume $\Psi_{-}(q ; \zeta)=\Psi_{+}(-q ; \zeta)$, therefore

$$
Q(\zeta, q)=\frac{\Psi_{+}(q ; \zeta) \Psi_{+}(-q ; \zeta)}{\omega(\zeta)}
$$

Lemma 6. Let the potential $V(x)$ be an even function. If $\Phi_{m}(q)=0$, then $Q\left(\lambda_{m}, q\right)=0$.

Proof. Let $\Phi_{m}(q)=0$. Then $\Phi_{m}(-q)=0$ because $\Phi_{m}(x)$ is either odd or even. It is known (see, e.g., [31]) that for some neighbourhood $U$ of $\lambda_{m}$ there exists a family $\Psi(q ; \zeta)$ which is analytic with respect to $\zeta \in U$, and such that $\Psi\left(q ; \lambda_{m}\right)=\Phi_{m}(q)$ and $\Psi(q ; \zeta)=\Psi_{+}(q ; \zeta)$ for $\zeta \neq \lambda_{m}$. Since the function $\omega(\zeta)$ has a simple zero at the point $\lambda_{m}$, then the statement follows from equality (23)

Lemma 6 implies the following refinement of Theorem 2.

Proposition 2. The following assertions hold:

(i) If $H_{0}$ is the Schrödinger operator, then the set $\sigma_{4}$ from Theorem 2 is empty.

(ii) Let the potential $V(x)$ be an even function. Then the following assertions take place:

(a) For $\mu \neq+\infty$ all the eigenvalues of the operator $H(q, \mu)$ are simple and belong either to the set $\sigma_{1}$ or to the set $\sigma_{3}$ from Theorem 2.

(b) For $\mu=+\infty$ the set $\sigma_{3}$ is empty, and all the eigenvalues $\lambda_{m}$ from the spectrum of $H(q, \mu)$ are doubly degenerate.

(c) $\lambda_{0}$ does not belong to the spectrum of all the operators $H(q, \mu)$, while every eigenvalue of the form $\lambda_{2 m+1}$ belongs to the spectrum of any operator $H(0, \mu)$.

Note that in the case of the Schrödinger.operator, the number of the singular points of each function $E_{n}(q)$ is finite and does not exceed $n$. Moreover, in this case it is easy to establish the following statement.

Proposition 3. If the potential $V(x)$ is a function of the class $C^{k}(0 \leq k \leq \infty)$ on some interval $(c, d)$, then the functions $E_{n}(q)$ belong to the class $C^{k+2}$ on the set of all non-singular points of this interval. If $V(x)$ is a real-analytic function on an interval $(c, d)$, then the functions $E_{n}(q)$ are real-analytic at all non-singular points of the interval as well as at all singular points of the kind (a) or (b). In particular, for $\mu \neq+\infty$ the functions $E_{k}(q)$ are real-analytic if the potential $V(x)$ is a real-analytic function.

Proof. For non-singular points the statement of the proposition immediately follows from formula (19) and the implicit function theorem. Let now $q_{0}$ be a singular point of the kind (a) for the function $E_{k}(q)$. We consider the function $\widehat{Q}_{\mu}(\zeta, q)$ defined by the equality

$$
\widehat{Q}_{\mu}(\zeta, q)=\omega(\zeta)\left(Q(\zeta, q)+\mu^{-1}\right)
$$


In some neighbourhood of the point $\left(\lambda_{k}, q_{0}\right)$ the function $\widehat{Q}_{\mu}$ is analytical, and in some neighbourhood of the point $q_{0}$ the function $E_{k}(q)$ is defined as an implicit function by the equation

$$
\widehat{Q}_{\mu}(E, q)=0
$$

It remains to verify that

$$
\frac{\partial \widehat{Q}_{\mu}}{\partial E}\left(\lambda_{m}, q_{0}\right) \neq 0
$$

We have

$$
\frac{\partial \widehat{Q}_{\mu}}{\partial E}=\left(\frac{\partial Q}{\partial E}\right) \omega(E)+\left(Q+\mu^{-1}\right) \omega^{\prime}(E) .
$$

Because $\left|\frac{\partial Q}{\partial E}\left(\lambda_{m}, q_{0}\right)\right|<\infty$, we have $\frac{\partial Q}{\partial E}\left(\lambda_{m}, q_{0}\right) \omega\left(\lambda_{m}\right)=0$. Since $\omega(\zeta)$ has a single zero in the point $\lambda_{m}$, then $\omega^{\prime}\left(\lambda_{m}\right) \neq 0$. Besides, $Q+\mu^{-1}$ does not vanish in the point $\left(\lambda_{m}, q_{0}\right)$. Thus relation (26) is proved. In the case of points of the kind (b) the proof is similar

As a corollary of Theorem 1 we obtain the following statement.

Proposition 4. For all $q \in \mathbb{R}$ the inequality

$$
-\frac{\mu^{2}}{4} \leq E_{0}(q)
$$

holds.

Proof. We denote by $B$ the selfajoint operator in $L^{2}(\mathbb{R})$ associated with the quadratic form $\hat{B}(\psi)=\left\|\psi^{\prime}\right\|^{2}+\mu|\psi(q)|^{2}$. It follows from Theorem 1 that $B \leq H$. The ground state of the operator $B$ is equal to $-\frac{\mu^{2}}{4}$ (see [2]). Hence we obtain (27)

Further we shall use the following hypothesis about the potential $V$ :

(V2) The set of all discontinuity points of the function $V(x)$ is finite (possibly, empty); $V(x)$ decreases on the half-line $x \leq 0$ and increases on the half-line $x \geq 0$. This function is differentiable at continuity points.

(V3) There exists a point $x_{0}>0$ such that the derivative $V^{\prime}(x)$ is a locally absolutely continuous function for $|x|>x_{0}$, and $\int_{|x|>x_{0}}\left|\frac{5 V^{\prime 2}(x)}{4 V^{3}(x)}-\frac{V^{\prime \prime}(x)}{V^{2}(x)}\right| \sqrt{V(x)} d x<\infty$.

For convenience, the partial derivatives of the function $Q(\zeta, q)$ with respect to $q$ are denoted by a prime: $\frac{\partial Q(\zeta, q)}{\partial q}=Q^{\prime}(\zeta, q)$, etc.

Lemma 7. Suppose that condition (V2) is fulfilled. Then the following statements are true:

(i) For all $\zeta \in \rho\left(H_{0}\right)$ the function $q \mapsto Q(\zeta, q)$ has the continuous second derivative $Q^{\prime \prime}(\zeta, q)$ at all the continuity points of the function $V(q)$; at the discontinuity points there exist the one-sided limits $Q^{\prime \prime}(\zeta, s-0)$ and $Q^{\prime \prime}(\zeta, s+0)$.

(ii) Let $E \in \mathbb{R}$ and $E \neq \lambda_{n}$ for every $n$. If $\Psi_{+}(s ; E) \neq 0$ and $Q^{\prime}(E, s)=0 \quad(s \in \mathbb{R})$, then $Q^{\prime \prime}(E, s \pm 0)<0$. 
(iii) Let $E \in \mathbb{R}$ and $E<\lambda_{0}$. There exists at most one point $q_{0} \in \mathbb{R}$ such that $Q^{\prime}\left(E, q_{0}\right)=0$. If $Q^{\prime}\left(E, q_{0}\right)=0$, then $Q^{\prime}(E, q)>0$ for $q>q_{0}$, and $Q^{\prime}\left(E, q_{0}\right)<0$ for $q<q_{0}$.

Proof. Assertion (i): If condition (V2) is fulfilled, then each solution of the equation $H_{0} F=\zeta F$ has piecewise continuous second derivative $F^{\prime \prime}$, and in each point $s \in \mathbb{R}$ there exist the one-sided limits $F^{\prime \prime}(s-0)$ and $F^{\prime \prime}(s+0)$. Therefore assertion (i) follows from equality (19).

Assertion (ii): Using equality (19), we obtain in a neighbourhood of $s$

$$
Q^{\prime}(E, q)=\frac{\left[\Psi_{+}^{\prime}(q ; E) \Psi_{-}(q ; E)+\Psi_{+}(q ; E) \Psi_{-}^{\prime}(q ; E)\right]}{\omega(E)},
$$

and

$$
\begin{aligned}
Q^{\prime \prime}(E, q) & =\frac{\Psi_{+}^{\prime \prime}(q ; E) \Psi_{-}(q ; E)+\Psi_{+}(q ; E) \Psi_{-}^{\prime \prime}(q ; E)+2 \Psi_{+}^{\prime}(q ; E) \Psi_{-}^{\prime}(q ; E)}{\omega(E)} \\
& =2 \frac{(V(q)-E) \Psi_{+}(q ; E) \Psi_{-}(q ; E)+\Psi_{+}^{\prime}(q ; E) \Psi_{-}^{\prime}(q ; E)}{\omega(E)}
\end{aligned}
$$

if $q$ is a continuity point of the function $V$. From the equality $Q^{\prime}(E, s)=0$ and equality (19) we obtain

$$
\Psi_{-}^{\prime}(q ; E)=-\frac{\Psi_{-}(q ; E) \Psi_{+}^{\prime}(q ; E)}{\Psi_{+}(q ; E)}+o(1) \quad \text { as } q \rightarrow s .
$$

Substituting this expression into (29), we obtain

$$
Q^{\prime \prime}(E, q)=2 \Psi_{-}(q ; E) \frac{(V(q)-E) \Psi_{+}^{2}(q ; E)-\Psi_{+}^{\prime 2}(q ; E)}{\omega(E) \Psi_{+}(q ; E)}+o(1)
$$

as $q \rightarrow s$. Denote

$$
f(q)=(V(q)-E) \Psi_{+}^{2}(q ; E)-\Psi_{+}^{\prime 2}(q ; E) .
$$

We have, at the continuity points $q$ of the function $V$,

$$
\begin{aligned}
f^{\prime}(q) & =V^{\prime}(q) \Psi_{+}^{2}(q ; E)+2(V(q)-E) \Psi_{+}(q ; E) \Psi_{+}^{\prime}(q ; E)-2 \Psi_{+}(q ; E) \Psi_{+}^{\prime \prime}(q ; E) \\
& =V^{\prime}(q) \Psi_{+}^{2}(q ; E)+2 \Psi_{+}^{\prime}(q ; E)\left[(V(q)-E) \Psi_{+}(q ; E)-\Psi_{+}^{\prime \prime}(q ; E)\right] \\
& =V^{\prime}(q) \Psi_{+}^{2}(q ; E) .
\end{aligned}
$$

Thus $f^{\prime}(q) \geq 0$ at the continuity points $q$ of the function $V$ obeying $q \geq 0$. Since $V(q)$ has positive jumps provided $q \geq 0$, the function $f(q)$ increases for $q \geq 0$. Now we shall prove that

$$
\liminf _{q \rightarrow+\infty} f(q) \leq 0
$$

Let us rewrite $f(q)$ in the form

$$
f(q)=g(q)-2 \Psi_{+}^{\prime 2}(q ; E) \quad \text { where } g(q)=(V(q)-E) \Psi_{+}^{2}(q ; E)+\Psi_{+}^{\prime 2}(q ; E) .
$$


To prove (32) it is sufficient to show that

$$
\liminf _{q \rightarrow+\infty} g(q) \leq 0
$$

In order to prove this, we show that $\int_{a}^{\infty} g(x) d x<+\infty$ for some $a>0$. Since the function $\Psi_{+}(\cdot ; E)$ is square integrable on the half-line $(0,+\infty)$ and $\Psi_{-}(\cdot ; E) \notin L^{2}(0,+\infty)$, then $\Psi_{+}\left(x_{1} ; E\right)<\Psi_{-}\left(x_{1} ; E\right)$ for some $x_{1}>0$. Similarly $\Psi_{+}\left(x_{2} ; E\right)>\Psi_{-}\left(x_{2} ; E\right)$ for some $x_{2}<0$. Therefore there exists $x_{0} \in\left[x_{2}, x_{1}\right]$ such that $\Psi_{+}\left(x_{0} ; E\right)=\Psi_{-}\left(x_{0} ; E\right)$. We define now an auxiliary function $h$ as

$$
h(x)= \begin{cases}\Psi_{+}(x ; E) & \text { for } x \geq x_{0} \\ \Psi_{-}(x ; E) & \text { for } x \leq x_{0} .\end{cases}
$$

It is easy to show that for any function $\varphi \in \mathcal{D}\left(H_{0}\right)$

$$
\left\langle h \mid\left(H_{0}-E\right) \varphi\right\rangle=-\gamma \varphi\left(x_{0}\right),
$$

where $\gamma$ is the jump of the function $h^{\prime}$ at the point $x_{0}$. From (34) and Theorem 1 we obtain that $h \in \mathcal{Q}\left(H_{0}\right)$, therefore the function $(V(x)-E) h^{2}(x)+h^{\prime 2}(x)$ belongs to the space $L^{1}(\mathbb{R})$. Hence $g \in L^{1}\left(x_{0}, \infty\right)$ and the relation (32) is proved. Since $f$ increases for $q \geq 0$, then $f(q) \leq 0$ for all $q \geq 0$. Let us suppose that $f(q)=0$ for all $q \geq q_{1}$. Without loss of generality we can assume that $V(q)$ is continuous for $q \geq q_{1}$. Then $V^{\prime}(q)=0$ for the same $q$ (see equality (31)), i.e. the function $V(q)$ is constant for $q \geq q_{1}$, but this contradicts to condition (V1). Thus $f(q)<0$ for all $q \geq 0$. Remember that for $E<\lambda_{0}$ the values of the functions $\Psi_{+}(q ; E), \Psi_{-}(q ; E)$ and $\omega(E)$ are strictly positive. Therefore for all points $s \geq 0$ statement (ii) follows from (30). For points $s<0$, we argue similarly.

(iii): It is sufficient to prove the uniqueness of the point $q_{0}$ for which $Q^{\prime}\left(E, q_{0}\right)=0$. Note that $q_{0}$ is an isolated zero of the function $Q^{\prime}(E, \cdot)$; otherwise, we have $Q^{\prime \prime}\left(E, q_{0}-\right.$ $0)=0$ or $Q^{\prime \prime}\left(E, q_{0}+0\right)=0$ with contradictory to statement (ii). Let $Q^{\prime}\left(E, q_{1}\right)=0$ where $q_{1}$ is such a point that $q_{1}>q_{0}$ and $Q^{\prime}(E, q) \neq 0$ if $q \in\left(q_{0}, q_{1}\right)$. Then $Q^{\prime \prime}\left(E, q_{0}+0\right)<0$ and $Q^{\prime \prime}\left(E, q_{1}-0\right)<0$ simultaneously, hence for sufficiently small $\delta>0$ we obtain that $Q^{\prime}\left(E, q_{0}+\delta\right)<0$ and $Q^{\prime}\left(E, q_{1}-\delta\right)>0$. Therefore $Q^{\prime}(E, q)=0$ for some $q \in$ $\left(q_{0}+\delta, q_{1}-\delta\right)$. This contradiction completes the proof

Lemma 8. Suppose that the conditions (V1) and (V3) are fulfilled. If $E \in \mathbb{R} \backslash$ $\sigma\left(H_{0}\right)$, then $Q(q, E) \rightarrow 0$ as $q \rightarrow \pm \infty$.

Proof. It is known that under condition (V3) the following asymptotics take place as $x \rightarrow+\infty$ (see $[15,32])$ :

$$
\begin{aligned}
& \Psi_{ \pm}(x ; \zeta)=(V(x)-\zeta)^{-\frac{1}{4}} \exp \left[\mp \int_{x_{0}}^{x} \sqrt{V(x)-\zeta} d x\right](1+o(x)) \\
& \Psi_{ \pm}(x ; \zeta)=(V(x)-\zeta)^{-\frac{1}{4}} \exp \left[ \pm \int_{x_{0}}^{x} \sqrt{V(x)-\zeta} d x\right](1+o(x)) .
\end{aligned}
$$

From this asymptotics we obtain that, for a fixed $E \in \mathbb{R}, E \neq \lambda_{n}$,

$$
Q(E, q) \sim V(q)^{-\frac{1}{2}} \quad \text { for } q \rightarrow \pm \infty .
$$

From (35) and assumption (V1) the lemma follows 
The following theorem is the main result of the paper.

Theorem 4. For the Schrodinger operator $H_{0}$ the functions $E_{k}(q)$ have the following properties:

(i) Let $\mu<0$ and $k \geq 1$. Then the function $E_{k}(q)$ attains the maximum $\lambda_{k}$ at those points $q \in \mathbb{R}$ for which $\Phi_{k}(q)=0$ and has no other points of local maximum. Besides, $E_{k}(q)>\lambda_{k-1}$ for all $q \in \mathbb{R}$.

(ii) Let $0<\mu<\infty$ and $k \geq 1$. Then the function $E_{k}(q)$ attains its minimum $\lambda_{k-1}$ at those points $q \in \mathbb{R}$ for which $\Phi_{k-1}(q)=0$ and has no other points of local minimum. Besides, $E_{k}(q)<\lambda_{k}$ for all $q \in \mathbb{R}$.

(iii) Let $\mu=\infty$ and $k \geq 1$. Then:

(a) $\lambda_{0}<E_{1}(q)$ for all $q \in \mathbb{R}$.

(b) The function $E_{1}(q)$ attains its maximum which is equal to $\lambda_{1}$ at that point $q$ for which $\Phi_{1}(q)=0$ and has no other points of local maximum.

(c) For $k \geq 2$ the function $E_{k}(q)$ attains its minimum $\lambda_{k-1}$ at those points $q \in \mathbb{R}$ for which $\Phi_{k-1}(q)=0$, and attains its maximum $\lambda_{k}$ at those points $q \in \mathbb{R}$ for which $\Phi_{k}(q)=0$; this function has no other points of local maximum or local minimum.

(iv) Let the conditions (V2) and (V3) are fulfilled. Then:

(a) If $0<\mu \leq \infty$, then $\lim _{q \rightarrow \pm \infty} E_{k}(q)=\lambda_{k-1}$ for $k \geq 1$.

(b) If $\mu<0$, then $\lim _{q \rightarrow \pm \infty} E_{k}(q)=\lambda_{k}$ for $k \geq 0$. For the same values of $\mu$, there exists a unique point $q_{0}$ such that $E_{0}^{\prime}\left(q_{0}\right)=0$, moreover, $E_{0}^{\prime}(q)>0$ if $q>q_{0}$, and $E_{0}^{\prime}(q)<0$ if $q<q_{0}$.

Proof. (i) - (iii): These assertions immediately follow from Theorem 3 and Lemma $7 /($ ii).

(iv): Let $\mu<0$; we shall consider the function $E_{k}(q)$. Take $E^{*} \in\left(\lambda_{k-1}, \lambda_{k}\right)$. By virtue of Lemma 8 there exists $q_{0} \in \mathbb{R}$ such that all points $q>q_{0}$ are non-singular ones for the function $E_{k}(q)$, and at these points

$$
Q\left(E^{*}, q\right)+\mu^{-1}<0
$$

Since $Q$ increases as a function of $E$, we obtain from (32) that $E^{*}<E_{k}(q)<\lambda_{k}$ for all $q>q_{0}$. Thus for $\mu<0$ we have $\lim _{q \rightarrow \pm \infty} E_{k}(q)=\lambda_{k}$. If $\mu>0$, we argue in a similar manner. Let now $\mu<0$. Then $-\frac{\mu^{2}}{4} \leq E_{0}(q)<\lambda_{0}$ for all $q$, therefore the function $E_{0}(q)$ has at least one minimum. From (10) we obtain

$$
E_{0}^{\prime}(q)=-Q^{\prime}\left(E_{0}(q), q\right)\left[\frac{\partial Q}{\partial E}\left(E_{0}(q), q\right)\right]^{-1}
$$

hence the reference to Lemma 7 completes the proof

Acknowledgments. The work is partly supported by RFFR and Russian Ministry of Education. 


\section{References}

[1] Albeverio, S., Fenstad, J. E., Høegh-Kron, R. and T. Lindstrøm: Nonstandard Methods in Stochastic Analysis and Mathematical Physics. New York et al.: Academic Press 1986.

[2] Albeverio, S., Gesztesy, F., Høegh-Krohn, R. and H. Holden: Solvable Models in Quantum Mechanics. Berlin et al.: Springer-Verlag 1988.

[3] Altarelli, M.: Electronic states in semiconductor superlatices and quantum wells: an overview. In: Springer Ser. Solid-State Sci. (Electronic materials) (eds.: I. R. Chelikowsky and A. Franciosi). Berlin et al.: Springer-Verlag 95 (1991), $215-232$.

[4] Altarelli, M. and G. Platero: Magnetic hole levels in quantum wells in parallel magnetic field. Surf. Sci. 196 (1988), $540-544$.

[5] Ando, T., Fauler, A. and F. Stern: Electronic properties of two-dimensional systems. In: Rev. Modern Phys. 54 (1982), 437 - 672.

[6] Bagwell, P. E. and R. K. Lake: Resonances in transmission through an oscillating barrier. Phys. Rev. B46 (1992), $15329-15336$.

[7] Beenakker, C. W. J. and H. van Houten: Quantum transport in semiconductor nanostructures. In: Solid State Phys., Advances in Res. and Appl. (eds.: H. Ehrenreich and D. Turnbull). Boston et al.: Acad. Press 44 (1991), $1-228$.

[8] De Dios Leyva, M. and V. Galindo: Interband optical absorption in superlattices in an in-plane magnetic field. Phys. Rev. B.48 (1993), 4518 - 4523.

[9] Dunford, N. J. T. and Schwartz: Linear operators. Part II: Spectral Theory. New York et al.: Interscience 1963.

[10] Gerard, G.-M. and J.-Y. Marzin: Optical study of probability densities in quantum well eigenstates. Surf. Sci. 229 (1990), $433-438$.

[11] Geyler, V. A. and I. V. Chudaev: The spectrum of a quasi-two-dimensional system in the presents of a parallel magnetic field (in Russian). Zhurn. Vych. Matem. i Matem. Fiz. 37 (1997)2, 214 - 222; Engl. transl. in: Comput. Math. and Math. Phys. 37 (1997), 210 $-218$.

[12] Geyler, V. A., Margulis, V. A. and I. I. Chuchaev: Zero-range potentials and Carleman operators (in Russian). Sibir. Matem. Zhurnal 36 (1995), 828 - 841; Engl. transl. in: Siberian Math. J. 36 (1995), 714 - 726.

[13] Geyler, V. A., Margulis, V. A. and I. V. Chudaev: Harmonic oscillator with a moving point perturbation (in Russian). Matem. Modelirovanie 7 (1995)5, p. 45.

[14] Gumbs, G.: Self-consistent density of states for a single- and double-quantum-well structure in a parallel magnetic field. Phys. Rev. B54 (1996), $11354-11358$.

[15] Hartman, P.: Ordinary Differential Equations. New York et al.: J. Wiley \& Sons 1964.

[16] Ho, T.-L.: Oscillatory tunneling between quantum Hall systems. Phys. Rev. B50 (1994), $4514-4533$.

[17] Krein, M. G. and H. K. Langer: On defect subspaces and generalized resolvents of an Hermitian operator in the space $\Pi_{\kappa}$. Funct. Anal. $\Lambda$ ppl. 5 (1971), $59-71$.

[18] Landau, L. D. and E. M. Lifshitz: Course in Theoretical Physics. Volume III: Quantum Mechanics. Oxford: Pergamon Press 1977.

[19] Leavitt, R. P.: Exactly solvable one-dimensional model for impurity binding energies in quantum wells. Phys. Rev. B37 (1988), $7118-7120$.

[20] Lee, H. R., Oh, H. G., George, T. F. and C. I. Um: Electron energy levels in a quantum well within an in-plane magnetic field. J. Appl. Phys. 66 (1989), $2442-2445$. 
[21] Levinson, Y. B. and E. V. Sukhorukov: Bending of electron edge states in a magnetic field. J. Phys.: Condensed Matter 3 (1991), 7291 - 7306.

[22] Maan, J. C.: Two-dimensional systems, heterostructures, and superlattices. In: Springer Ser. Solid-State Sci. 53 (1984), 183 - 191.

[23] Maan, J. C.: Magneto-optical properties of superlattices and quantum wells. Surf. Sci. 196 (1988), $518-532$.

[24] Maan, J. C.: Optical studies of tunneling in a magnetic field. Physica B 201 (1994), 391 $-396$.

[25] Montambaux, G. and P. B. Littlewood: "Fractional" quantum Hall effect in a quasi-onedimensional conductor. Phys. Rev. Lett. 62 (1989), 953 - 956.

[26] Naimark, M. A.: Linear Differential Operators. New-York: Ungar 1968.

[27] Niu, Q. and D. J. Thouless: Quantum Hall effect with realistic boundary conditions. Phys. Rev. B35 (1987), $2188-2197$.

[28] Pavlov, B. S.: The theory of extensions and explicitly solvable models. Russian Math. Surveys 42 (1987), $99-131$.

[29] Pimpale, A., Holloway, S. and R. J. Smith: Tunneling through moving barriers. J. Phys. A24 1991, 3533 - 3550.

[30] Prange, R. and S. M. Girvin: The Quantum Hall Effect. New-York et al.: Springer-Verlag 1987.

[31] Smith, C. G. et al.: Transport in a superlattice of $1 D$ ballistic channels. J. Phys.: Condens. Matter 2 (1987), 3405-3414.

[32] Titchmarsh, E. C.: Eigenvalues Expansions Associated with Second-Order Differential Euations. Oxford: Clarendon Press 1946.

[33] Zheng, $\mathrm{H}$. and $\mathrm{H}$. Zhou: Influence of a parallel magnetic field on localization of disordered two-dimensional electrons in $\mathrm{GaAs} / \mathrm{Al}_{x} \mathrm{Ga}_{1-x}$ As heterostructures. Phys. Rev. B41 (1990), $1140-1143$. 